\title{
Genetic variation of rs3811463 is associated with gestational diabetes mellitus susceptibility
}

\author{
YUN LIU ${ }^{1}$, ZHI-PING GE ${ }^{2}$, LI-ZHOU SUN ${ }^{2}$, PEI TONG ${ }^{1}$ and HONG-MEI LU ${ }^{2}$ \\ ${ }^{1}$ Department of Geriatric Endocrinology, The First Affiliated Hospital of Nanjing Medical University; \\ ${ }^{2}$ Department of Obstetrics, The First Affiliated Hospital of Nanjing Medical University, \\ Jiangsu Women and Children Health Hospital, Nanjing, Jiangsu 210029, P.R. China
}

Received March 4, 2017; Accepted August 24, 2017

DOI: $10.3892 / \mathrm{etm} .2017 .5188$

\begin{abstract}
Gestational diabetes mellitus (GDM) is a growing health concern, and it increases the risk of adverse pregnancy outcomes with substantial long-term adverse health impacts on mothers and their offspring. Several studies have revealed specific associations between genetic variants and the risk of GDM. Single nucleotide polymorphisms (SNPs) are the major type of genetic variation in humans. Let-7 microRNA targets are enriched for genes containing SNPs associated with glucose metabolism, including Lin28. In the present study, the effect of T/C variants of rs3811463 (a SNP located near to the let-7 binding site in Lin28) on GDM risk was investigated. A GDM rat model was successfully constructed using a high fat diet and streptozotocin injection, and the primary skeletal muscle cells were isolated. The cell transfection results demonstrated that $\mathrm{rs} 3811463-\mathrm{T} / \mathrm{C}$ significantly affected the glucose uptake and insulin sensitivity. Reverse transcription-quantitative polymerase chain reaction analysis indicated that the $\mathrm{C}$ allele at rs3811463 regulated the expression of glucose metabolism-associated genes insulin-like growth factor two binding protein 2 and glucokinase. Western blot analysis data revealed that replacement of the $\mathrm{T}$ allele by the $\mathrm{C}$ allele at rs 3811463 modulated the protein level of Sirtuin 1. Taken together, it was concluded that the let-7/Lin 28 axis regulated glucose uptake and insulin sensitivity by modulating the expression of glucose metabolism-associated proteins. These findings provide novel
\end{abstract}

Correspondence to: Dr Hong-Mei Lu, Department of Obstetrics, The First Affiliated Hospital of Nanjing Medical University, Jiangsu Women and Children Health Hospital, 368 North-Jiangdong Road, Nanjing, Jiangsu 210029, P.R. China

E-mail: lu_hongmei01@163.com

Abbreviations: GDM, gestational diabetes; SNP, single nucleotide polymorphism; miRNA, microRNA; let-7, Lethal-7; T2DM, type 2 diabetes mellitus; STZ, streptozotocin; qRT-PCR, quantitative reverse transcription-PCR; GCK, glucokinase; IGF2BP2, insulin-like growth factor two binding protein 2; IRS1, Insulin receptor substrate 1

Key words: Lin28, Lethal-7, gestational diabetes, single nucleotide polymorphism evidence on the association between genetic variations of rs3811463 and GDM susceptibility.

\section{Introduction}

Gestational diabetes mellitus (GDM) is a common complication of pregnancy associated with an increased incidence of pregnancy complications, adverse pregnancy outcomes, and maternal and fetal risks of chronic health conditions later in life (1). The occurrence of GDM is a multi-factor and multi-stage process, which is directed by the environmental, dietary or genetic factors. However, the specific mechanism has not been fully elucidated. Well-documented risk factors for GDM include pre-pregnancy overweight and obesity (2). But some reports also showed that only a few people suffered from GDM under the context of the same diet, suggesting that different individuals displayed different susceptibility to food habits (3). Several studies referred to the close association between genetic variants and the risk of GDM (4).

Single nucleotide polymorphisms (SNPs) are the major type of genetic variation in human beings. Many studies reported that allelic variations of SNPs can influence the expression or function of their hosting genes. Increasing evidence revealed that microRNA (miRNA)-related SNPs significantly altered the biogenesis and/or function of the corresponding miRNA (5). miRNA is a small non-coding single-stranded RNA consisting of 18-24 nucleotides, widespread throughout of human genome (6). They typically regulate genes by binding to the $3^{\prime}$ UTR of the target mRNA, which destabilizes the mRNA and prevents translation (7). miRNAs are emerging as agents of metabolic and malignant regulation in development and disease. Lethal-7 (let-7) miRNA has gained notoriety owing to its regulation of stem cell differentiation and essential role in normal development (8). Let-7 miRNA cooperates with multiple transcription factors and directly regulates the targeted gene expression. A RNA binding protein and stem cell marker Lin28 is a main regulator of let-7, and is initially found in C.elegans to regulate the fate of cell both in early development and terminal differentiation (9). There is a double-negative feedback loop between Lin 28 and let-7: let-7 can repress the post-transcriptional translation of $\operatorname{Lin} 28$ (8), while Lin28 blocks the maturation of let-7 miRNA (10). 
Meta-analysis of published data confirmed that let-7 targets are enriched for gene containing SNPs associated with type 2 diabetes mellitus (T2DM) and control of fasting glucose in humans (11). A SNP located near the let-7 binding site in Lin28, the $\mathrm{T} / \mathrm{C}$ variants of rs3811463 could lead to differential regulation of Lin 28 by let-7 (12). The rs3811463 polymorphism in the let-7/Lin28 pathway significantly increased the risk of T2DM(13). Insulin resistance is the major determinant of T2DM and GDM (14). So there might be some relationship between genetic polymorphisms in the let/Lin28 pathway and the risk of GDM.

In our study, we constructed GDM rat model via high fat diet and STZ injection and then examined the body weight and blood glucose level. The primary skeletal muscle cells were successfully incubated and transfected with T/C variants of rs3811463 and then glucose uptake and insulin sensitivity were examined. Furthermore, the glucose metabolism-related and insulin sensitivity-related genes were detected after various recombined vectors transfection. Taken together, we found that C allele of rs3811463 mitigated the expression of let-7, regulated the expression of glucose metabolism-related and insulin sensitivity-related genes to participate in the development of GDM.

\section{Materials and methods}

Construction of GDM rats model. Total 40 adult SPF Wistar rats, weighing 190-210 g, 7-8 weeks old, were purchased from Shanghai Silaike Laboratory Animal Limited Liability Company (Shanghai, China). Rats were kept at standard laboratory conditions at $18-25^{\circ} \mathrm{C}$ with $12 \mathrm{~h}$ light: $12 \mathrm{~h}$ dark. Standard rodent feed and water were available ad libitum. All procedures were performed in accordance with the Guide for the Humane Use and Care of Laboratory. This study was approved by the Committees of The First Affiliated Hospital with Nanjing Medical University and Jiangsu Women and Children Health Hospital. After a week of adaptation, the female and male rats were mated at the ratio of 2:1. Next morning, the female vaginal suppository was checked and the pregnant rats were housed individually. The pregnant rats were fed with a high fat diet (HFD, $58 \%$ fat, $25 \%$ protein and $17 \%$ carbohydrate, as a percentage of total kcal) for an initial period of two weeks (15). Thereafter, rats received a $60 \mathrm{mg} / \mathrm{kg}$ intraperitoneal injection of streptozotocin (STZ) in freshly prepared sodium citrate buffer (0.1 mM, pH 4.5). The normal control (NC) group was administrated with the same amount of sodium citrate buffer. Rats with blood glucose levels after $72 \mathrm{~h}$ of STZ injection higher than $16.67 \mathrm{mM}$ were diagnosed as GDM for further experiments.

Isolation of primary cells. STZ-induced diabetic female rats were anaesthetizes with injection of pentobarbital $(60 \mathrm{mg} / \mathrm{kg})$ into abdominal cavity. The musculi quadriceps femoris of hind legs were isolated and washed with sterile PBS buffer to remove the connective tissues. Then the isolated tissues were sheared and digested with collagenase IA (Sigma, USA) at $4^{\circ} \mathrm{C}$ overnight. After centrifugation, skeletal muscle cells were cultured with Dulbecco modified Eagle medium (DMEM, GIBCO, USA) supplemented with $10 \%$ fetal bovine serum (FBS, GIBCO, USA) and $1 \%$ myllicin in a humidified atmosphere of $5 \% \mathrm{CO}_{2}$ at $37^{\circ} \mathrm{C}$.

Recombined vectors construction. Genomic DNA was extracted from the primary skeletal muscle cells using a
DNA extraction Kit (Invitrogen, USA). An 885 bp fragment of the 3' UTR of rat Lin28 gene centering rs3811463 and the predicted complementary site of let-7 were inserted into pEF6/V5-His-TOPO vector. A mutant plasmid with $\mathrm{C}$ allele at the site of rs3811463 was constructed using a site-directed mutagenesis kit (Stratagene, La Jolla, CA, USA). All recombined plasmids were verified by direct DNA sequencing. The wild type recombinant and mutated vectors were named pEF6/V5-His-TOPO: rs3811463T and pEF6/V5-His-TOPO: rs3811463C, respectively.

Cell transfection. The third passage skeletal muscle cells were seeded into 6 -well plates at the concentration of $1-2 \times 10^{6}$ cell/well. When the density reached $80-90 \%$, cell transfection was performed according to the manufacturer's instructions. About $0.8 \mu \mathrm{g}$ plasmid DNA or $2 \mu \mathrm{l}$ Lipofectamine 3000 was diluted with $50 \mu \mathrm{l}$ Opti-MEM FBS-free DMEM, respectively. After $5 \mathrm{~min}$ at room temperature, the diluted DNA and Lipofectamine 3000 were mixed gently. About $20 \mathrm{~min}$ later at room temperature, about $100 \mu \mathrm{l}$ of mixture was added into the cells and cultured at $37^{\circ} \mathrm{C}$ incubators. After $6 \mathrm{~h}$, the medium was replaced with fresh DMEM containing $10 \%$ FBS.

Glucose uptake and insulin sensitivity assay. Muscle cells were pretreated in low-glucose DMEM for $12 \mathrm{~h}$, and then transfected with pEF6/V5-His-TOPO, pEF6/V5-His-TOPO: rs3811463T or pEF6/V5-His-TOPO: rs3811463C, respectively. After $48 \mathrm{~h}$, the old medium was removed, and cells were incubated with $4 \mathrm{ml}$ of HEPES-buffer saline containing $\left[{ }^{3} \mathrm{H}\right]$-2-deoxy-D-glucose $(1.0 \mathrm{mCi} / \mathrm{ml})$ for $30 \mathrm{~min}$. After being washed with PBS, cells were trypsinized with $0.25 \%$ trypsin (Sigma, USA). The radioactivity was determined by scintillation counting. All experiments were assayed in at least three different independent repeat. For insulin sensitivity assay, cells were pretreated with $10 \mu \mathrm{g} / \mathrm{ml}$ of insulin for $4 \mathrm{~h}$, and then glucose uptake was detected according to the above protocol.

Quantitative reverse transcription- $P C R$ ( $q R T-P C R)$. Total RNA was extracted from muscle cells with Trizol reagent (Sigma, USA) in accordance with the manufacturer's instructions. The concentration and quality was measured by Nanodrop 2000 (Thermo Fisher Scientific, Wilmington, DE, USA). About $1 \mu \mathrm{g}$ of total RNA was reverse transcribed with SuperScript ${ }^{\circledR}$ III First Strand kit (Invitrogen, USA) according to the manufacturer's protocol. QRT-PCR was performed using TaqManGene Expression MasterMix (Thermo, USA). The parameters for qRT-PCR assay were as follows: $95^{\circ} \mathrm{C}$ for $10 \mathrm{~min}, 40$ cycles of $95^{\circ} \mathrm{C}$ for $15 \mathrm{sec}, 60^{\circ} \mathrm{C}$ for $1 \mathrm{~min}$. QRT-PCR was performed in triplicate with Applied Biosystems 7500 PCR System (Applied Biosystems, USA) according to the standard protocol. The mRNA levels of IRS1, IGF2BP2 and GCK genes were normalized to the level of GAPDH using the method of $2^{-\Delta \Delta C T}$ and were represented as fold induction. The primer sequences for qRT-PCR are listed in Table I.

Western blot analysis. After various treatments, muscle cells were harvested and lysed with RIPA buffer (Sigma, USA). The concentration of protein was detected with bicinchoninic acid kit (Pierce, Rockford, IL). Proteins (40 $\mu \mathrm{g} /$ lane) were subjected into $12 \%$ SDS-polyacrylamide gel and then 
transferred onto a polyvinylidene fluoride (PVDF) membrane (Millipore, Bedford, MA, USA). Subsequently, the membrane was blocked with $2 \%$ non-fat milk in TBS containing $0.05 \%$ Tween-20 (TBST) for $1 \mathrm{~h}$ at room temperature and incubated overnight at $4^{\circ} \mathrm{C}$ with the primary antibodies against Sirtuin 1 (SIRT1, Abcam, USA). After being washed with TBST for five times, the membranes were probed with horseradish peroxidase-conjugated secondary antibodies (1:10,000 dilution, zhongshan, Beijing, China). The membranes were developed with the enhanced chemiluminescence kit (Amersham). Relative band intensity of SIRT1 was detected with Quantity One v4.62 (Bio-Rad, USA) and normalized to GAPDH.

Statistical analysis. Statistical analysis in the present study was performed with Graphpad prism 6.0 (GraphPad Software, La Jolla, CA, USA). All data from the experiments are presented as the mean \pm standard deviation. We used one-way analysis of variance (ANOVA) to determine significance between two groups via SPSS software (version 16.0, SPSS Inc, Chicago, Illinois). The letters in the Figures indicate the significant difference $(\mathrm{P}<0.05)$ in various treatments by one-way ANOVA.

\section{Results}

GDM rat model was successfully constructed by high fat diet and STZ injection. In the experiment, we selected 13 of pregnant female rats for model construction. To verify the effect of high fat diet and STZ injection, we examined the body weight and blood glucose of tail vein at the 3rd, 6th, 9th and 12th d after STZ injection shown in Table II. There was no significant difference on the body weight at the $3 \mathrm{rd}$ and 6 th $\mathrm{d}$ between NC and GDM groups ( $\mathrm{P}>0.05)$. However, at the 9th and 12th d, the body weight of GDM was obviously lower than that in NC group $(\mathrm{P}<0.05)$. Meanwhile, the blood glucose in GDM group was significantly higher than that in NC group at the 3rd, 6th, 9th and 12th $\mathrm{d}(\mathrm{P}<0.05)$. Thus we concluded that GDM rat model was successfully constructed for follow-up experiments.

The effect of replacement of the $T$ allele by the $C$ allele at rs3811463 on glucose uptak. To investigate the effect of replacement of the $\mathrm{T}$ allele by the $\mathrm{C}$ allele at rs3811463 on glucose uptake, we transfected muscle cells with the wild and mutated vectors and performed ${ }^{3} \mathrm{H}$ glucose uptake experiments in Fig. 1A. In pEF6/V5-His-TOPO-transfected cells (Ctrl group), the radioactivity of lipid extraction (132 $\pm 14 \mathrm{CPM} /$ flask) was lower than that in rs3811463-T $(152 \pm 31 \mathrm{CPM} / \mathrm{flask})$ and rs3811463-C $(188 \pm 20 \mathrm{CPM} /$ flask $)$ groups $(\mathrm{P}<0.05)$. And the radioactivity of lipid extraction after rs3811463-C transfection was higher than that in rs3811463-T-transfected group $(\mathrm{P}<0.05)$. These data showed that the overexpression of Lin28 enhanced the activity of glucose uptake, and the replacement of the $\mathrm{T}$ allele by the $\mathrm{C}$ at rs 3811463 promoted the glucose uptake.

To test the insulin sensitivity, cells were pretreated with insulin for $4 \mathrm{~h}$, and then transfected with various vectors for glucose uptake detection. In Fig. 1B, the radioactivity of lipid extraction in pEF6/V5-His-TOPO-transfected cells (Ctrl group) was $147 \pm 5 \mathrm{CPM} /$ flask, which was significantly lower than that in rs3811463-T (167 $\pm 22 \mathrm{CPM} /$ flask) and rs3811463-C (215 \pm 17 $\mathrm{CPM} /$ flask) groups $(\mathrm{P}<0.05)$. What's more, rs3811463-C
Table I. Primers for qRT-PCR.

\begin{tabular}{lll}
\hline Genes & Forward primers (5'-3') & Reverse primers (5'-3') \\
\hline IRS1 & cgatggcttctcagacgtg & cagccegcttgttgatgttg \\
IGF2BP2 & gactaccccgaccagaactg & gaggcgggatgttccgaatc \\
GCK & accaagcggtatcagcatgtg & tggacttctctgtgattggca \\
GAPDH & tgtgggcatcaatggatttgg & acaccatgtattccgggtcaat \\
\hline
\end{tabular}

transfection increased the radioactivity than rs3811463-T. These results showed that the overexpression of Lin 28 enhanced the activity of glucose uptake after insulin stimulation, and the replacement of the $\mathrm{T}$ allele by the $\mathrm{C}$ at rs 3811463 promoted the glucose uptake after insulin stimulation.

$C$ allele at rs3811463 affected the expression of IGF2BP2 and $G C K$. To examine the mechanism by which $\mathrm{C}$ allele at rs3811463 modulated the glucose uptake, we analyzed the expression of glucose metabolism-related gene by qRT-PCR in Fig. 2. The transfection of rs3811463-T obviously upregulated the expression of let-7, but had no effect on the mRNA levels of in glucokinase (GCK), insulin-like growth factor two binding protein 2 (IGF2BP2) and insulin receptor substrate 1 (IRS1) genes. The rs3811463-C transfection had no effect on the levels of let-7 and IRS1, but significantly improved the expression of IG2BP2 and GCK. These data showed that the transfection of rs3811463 modulated the ability of glucose uptake by affecting the expression of glucose metabolism-related genes.

$C$ allele at rs3811463 modulated the expression of SIRT1. To explore the reason of $\mathrm{rs} 3811463-\mathrm{T} / \mathrm{C}$ transfection affecting the glucose uptake after insulin stimulation, we examined the expression of insulin sensitivity-related gene, SIRT1. SIRT1 is a conserved protein NAD+-dependent deacylases and thus the function is intrinsically linked to cellular metabolism (16). In Fig. 3, compared with pEF6/V5-His-TOPO-transfected cells (Ctrl group), the transfection of rs3811463-T and rs3811463-C significantly enhanced the protein level of SIRT1. But the rs3811463-C transfection resulted into the higher expression of SIRT1 than rs3811463-T. These data showed that $\mathrm{C}$ allele at rs3811463 modulated the insulin sensitivity by regulating the expression of SIRT1.

\section{Discussion}

In this study, we investigated the association between $\mathrm{T} / \mathrm{C}$ variants of rs3811463 in the let-7/Lin28 pathway and the risk of GDM. We injected pregnant female rats fed on HFD with STZ to induce GDM, which is a classic method for GDM induction (17). STZ injection improved the body weight and blood glucose, suggesting that GDM model was successfully constructed for further investigation. The replacement of the $\mathrm{T}$ allele by $\mathrm{C}$ allele of rs3811463 affected glucose uptake, the expression of let-7, glucose metabolism-related and insulin sensitivity-related genes for GDM. These findings revealed that genetic variations of rs3811463 may lead to altered status of the let-7/Lin28 loop and are associated with GDM susceptibility. 
Table II. The examination of body weight and blood glucose level.

Time (d)

\begin{tabular}{lcccc}
\cline { 2 - 4 } Group & 3rd & 6th & 9 th & 12th \\
\hline Body weight $(\mathrm{g})$ & & & & \\
NC & $171.12 \pm 2.6$ & $184.95 \pm 1.5$ & $203.08 \pm 5.1$ & $234.48 \pm 3.4$ \\
GDM & $174.00 \pm 3.7$ & $185.74 \pm 0.8$ & $180.71 \pm 2.8^{\mathrm{a}}$ & $191.58 \pm 2.1^{\mathrm{a}}$ \\
Blood glucose $(\mathrm{mM})$ & & & & \\
NC & $17.69 \pm 0.9$ & $17.26 \pm 1.9$ & $17.55 \pm 3.3$ & $17.39 \pm 1.7$ \\
GDM & $21.17 \pm 1.4^{\mathrm{a}}$ & $27.59 \pm 2.9^{\mathrm{a}}$ & $35.35 \pm 0.7^{\mathrm{a}}$ & $40.51 \pm 0.8^{\mathrm{a}}$ \\
\hline
\end{tabular}

${ }^{\mathrm{a}} \mathrm{P}<0.01$, compared with $\mathrm{NC}$. NC, negative control; GDM, gestational diabetes mellitus.

A

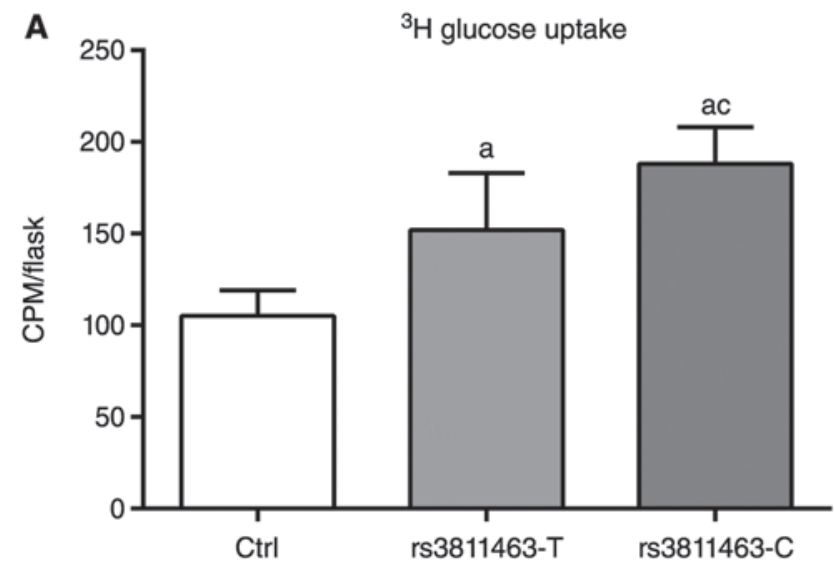

B

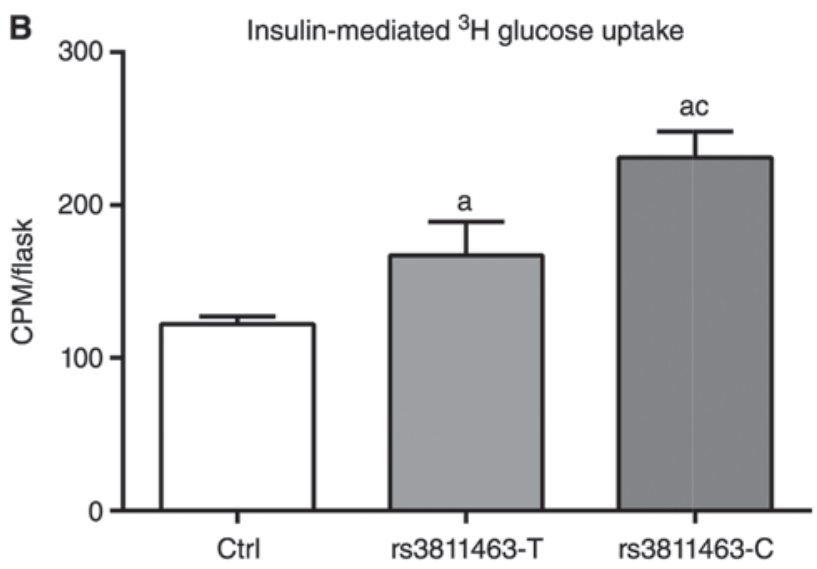

Figure 1. The effect of genetic variations of rs3811463 on glucose uptake and insulin sensitivity. (A) glucose metabolism assay after cell transfection. Muscle cells were pretreated in low-glucose DMEM for $12 \mathrm{~h}$, and then transfected with pEF6/V5-His-TOPO, pEF6/V5-His-TOPO: rs3811463T or pEF6/V5-His-TOPO: rs3811463C, respectively. The radioactivity was determined by scintillation counting. (B) insulin sensitivity analysis after cell transfection. For insulin sensitivity assay, cells were pretreated with $10 \mu \mathrm{g} / \mathrm{ml}$ of insulin for $4 \mathrm{~h}$, and then glucose uptake was performed. All experiments were assayed in triplicate and performed at least four times. Ctrl group, pEF6/V5-His-TOPO-transfected cells; rs3811463T, pEF6/V5-His-TOPO: rs3811463T-transfected cells; rs3811463C, pEF6/V5-His-TOPO: rs3811463C-transfected cells. The letters a and c showed the significant difference $(\mathrm{P}<0.05)$ compared with Ctrl group or rs3811463T group, respectively.

Previous studies showed that the highly conserved miRNA let-7 represses the post-transcriptional translation of Lin28, and Lin28 in turn blocks the maturation of let-7, forming a double-negative feedback loop (18). The Lin28/let-7 axis is highly conserved across the animal kingdom in organisms as evolutionarily distant as nematode worms and humans (19). Let-7 family of miRNAs contributed to the control of glucose homeostasis and insulin sensitivity, and the knockdown of the let-7 could be taken as a potential treatment for T2DM (20). Lin28A and Lin28b transgenic animals are more sensitive to insulin and have reduced peripheral glucose levels (19), which suggest that let-7/Lin 28 axis regulates glucose metabolism. In our study, we found that the overexpression of Lin 28 improved the ability of glucose uptake and promoted the expression of let-7. Our data provided new evidence that let-7/Lin28 pathway was involved in glucose metabolism, which also provided a new target for DM therapy.

Increasing evidence indicated the association between genetic variation and the risk of GDM (21). SNPs are the major type of human genomic variation. Among Han Chinese woman, three SNPs (rs4659441, rs3811463 and rs6697410) were detected in the 3'UTR of Lin28, and allelic variation of rs3811463 influenced let-7-related regulation of Lin28 expression (12). Previous studies showed that insulin resistance and defects in insulin secretion play key roles in the etiology of both GDM and T2DM. So we guess that there might be some link between rs3811463 and GDM susceptibility. In our study, we found that the replacement of $\mathrm{T}$ allele by $\mathrm{C}$ allele significantly modulated the glucose uptake and insulin sensitivity in GDM model, which revealed that genetic variations of rs3811463 were associated with the risk of GDM by disturbing the double-negative feedback loop. This might be the main reason for the association between gene polymorphism of let-7/Lin28 and GDM susceptibility. What's more, owing to the disturbance of the double-negative feedback loop, we found that $\mathrm{C}$ allele of rs3811463 improved the expression of Lin28 to suppress the level of let-7.

It is indicated some significant associations of GDM with SNPs in GCK, IGF2BP2 and IRS1 genes (21), which are thought to modulate pancreatic islet $\beta$-cell function (22). In our study, we found that $\mathrm{T}$ allele of rs3811463 had no significant effect 

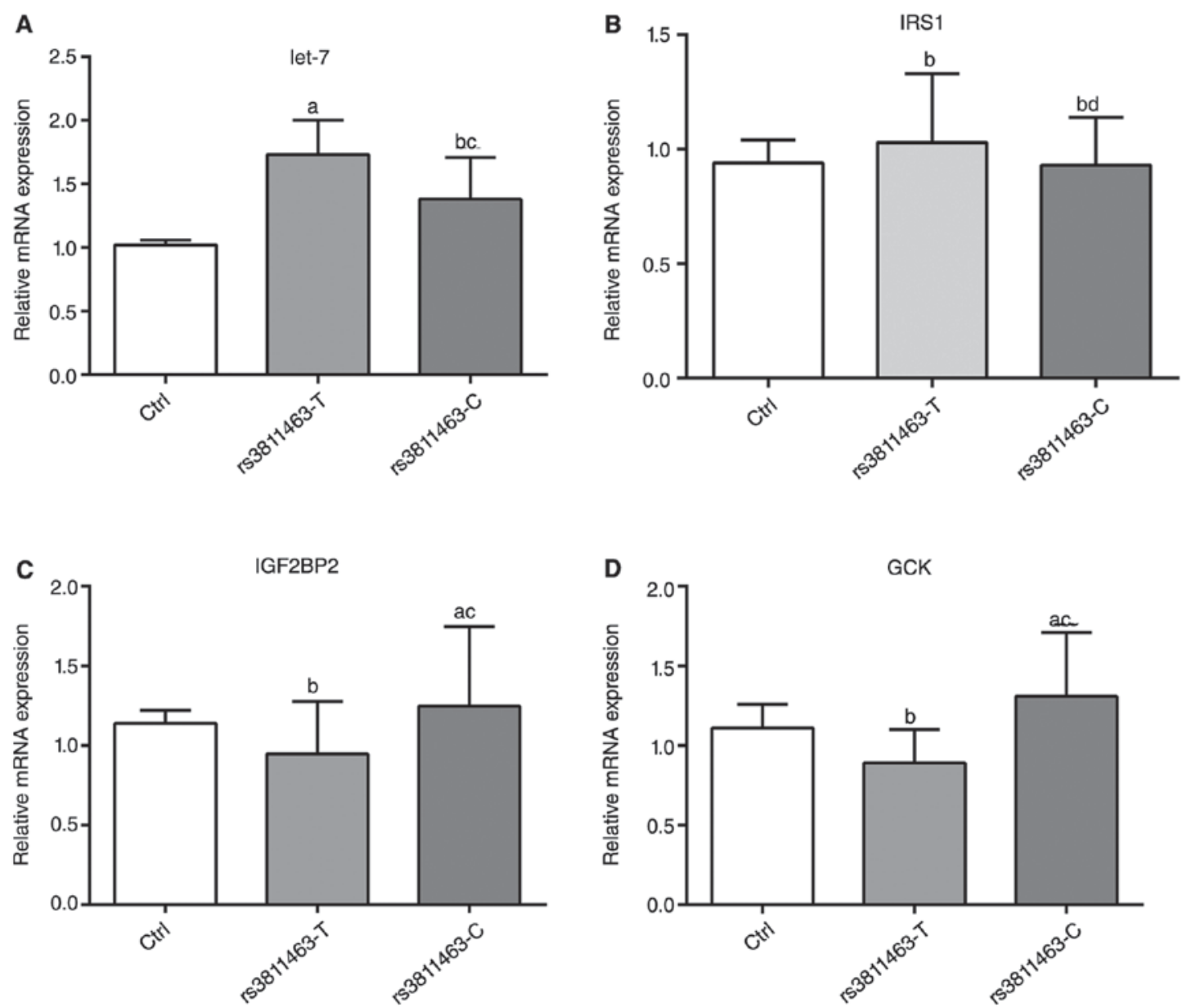

Figure 2. The mRNA levels of let-7 (A), IRS1 (B), IGF2BP2 (C) and GCK (D) after cell transfection determined by qRT-PCR. Muscle cells were pretreated in low-glucose DMEM for $12 \mathrm{~h}$, and then transfected with pEF6/V5-His-TOPO (Ctrl group), pEF6/V5-His-TOPO: rs3811463T (rs3811463T group) or pEF6/V5-His-TOPO: rs3811463C (rs3811463C group), respectively. After 48 h, RNA was isolated for qRT-PCR. GAPDH was used as the internal control. All experiments were performed in triplicate independent repeat analyzed with one-way ANOVA. Compared with Ctrl group, ${ }^{\mathrm{a}}$ means $\mathrm{P}<0.05,{ }^{\mathrm{b}}$ means $\mathrm{P}>0.05$. Compared with rs3811463T group, ${ }^{\mathrm{c}} \mathrm{P}<0.05,{ }^{\mathrm{d}} \mathrm{P}>0.05$.
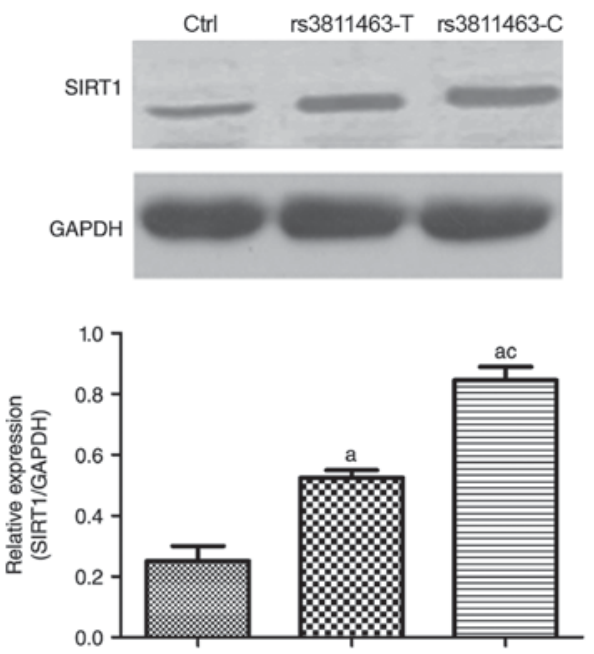

Figure 3. The relationship between genetic variation of rs 3811463 and SIRT1 protein level. Muscle cells were pretreated in low-glucose DMEM for $12 \mathrm{~h}$ before $4 \mathrm{~h}$ of $10 \mu \mathrm{g} / \mathrm{ml}$ of insulin treatment, and then transfected with pEF6/V5-His-TOPO (Ctrl group), pEF6/V5-His-TOPO: rs3811463T (rs3811463T group) or pEF6/V5-His-TOPO: rs3811463C (rs3811463C group), respectively. Cells were collected and protein was extracted with RIPA buffer for western blot. All experiments were carried out in triplicate independent repeat. All data were analyzed by one-way ANOVA. Compared with Ctrl group, ${ }^{\mathrm{a}} \mathrm{P}<0.05$; Compared with rs $3811463 \mathrm{~T}$ group, ${ }^{\mathrm{C}} \mathrm{P}<0.05$. on the levels of IRS1, IGF1BP2 and GCK, however, C allele of rs3811463 improved the expression of above three genes, suggesting that genetic variation of rs 3811463 was involved in the regulation of glucose-metabolism related genes. These results revealed the relationship between genetic variation of rs3811463 and glucose metabolism. SIRT1 is implicated in the regulation of mitochondrial function, energy metabolism, and insulin sensitivity (23). In our subject, we observed that $\mathrm{T}$ allele of rs 3811463 enhanced the protein level of SIRT1 and the replacement of $\mathrm{T}$ allele by $\mathrm{C}$ allele obviously improved the level of SIRT1. These data showed that let-7/Lin28 pathway regulated insulin sensitivity by modulating the expression of SIRT1. Taken together, let-7/Lin28 axis is a key factor for glucose metabolism and insulin sensitivity by regulating glucose metabolism-related and insulin sensitivity related genes. These findings revealed that gene polymorphism of let-7/Lin28 might be a new target for GDM susceptibility study.

\section{Acknowledgements}

The present study was supported by Jiangsu Province, maternal and child health research projects gynecological science key projects (F201406). 


\section{References}

1. Russo LM, Nobles C, Ertel KA, Chasan-Taber L and Whitcomb BW: Physical activity interventions in pregnancy and risk of gestational diabetes mellitus: A systematic review and meta-analysis. Obstet Gynecol 125: 576-582, 2015.

2. Zhang $\mathrm{C}$ and Ning Y: Effect of dietary and lifestyle factors on the risk of gestational diabetes: Review of epidemiologic evidence. Am J Clin Nutr 94 (6 Suppl): 1975S-1979S, 2011.

3. Bowers K, Tobias DK, Yeung E, Hu FB and Zhang C: A prospective study of prepregnancy dietary fat intake and risk of gestational diabetes. Am J Clin Nutr 95: 446-453, 2012.

4. Donin AS, Nightingale CM, Owen CG, Rudnicka AR, Jebb SA, Ambrosini GL, Stephen AM, Cook DG and Whincup PH: Dietary energy intake is associated with type 2 diabetes risk markers in children. Diabetes Care 37: 116-123, 2014.

5. Nicoloso MS, Sun H, Spizzo R, Kim H, Wickramasinghe P, Shimizu M, Wojcik SE, Ferdin J, Kunej T, Xiao L, et al: Single-nucleotide polymorphisms inside microRNA target sites influence tumor susceptibility. Cancer Res 70: 2789-2798, 2010.

6. Bartel DP: MicroRNAs: Target recognition and regulatory functions. Cell 136: 215-233, 2009.

7. Shukla GC, Singh J and Barik S: MicroRNAs: Processing, maturation, target recognition and regulatory functions. Mol Cell Pharmacol 3: 83-92, 2011.

8. Nguyen LH and Zhu H: Lin28 and let-7 in cell metabolism and cancer. Transl Pediatr 4: 4-11, 2015.

9. Sun X, Liu J, Xu C, Tang SC and Ren H: The insights of Let-7 miRNAs in oncogenesis and stem cell potency. J Cell Mol Med 20: 1779-1788, 2016.

10. Viswanathan SR, Daley GQ and Gregory RI: Selective blockade of microRNA processing by Lin28. Science 320 : 97-100, 2008

11. Zhu H, Shyh-Chang N, Segrè AV, Shinoda G, Shah SP, Einhorn WS, Takeuchi A, Engreitz JM, Hagan JP, Kharas MG, et al: The Lin28/let-7 axis regulates glucose metabolism. Cell 147: 81-94, 2011.

12. Chen AX, Yu KD, Fan L, Li JY, Yang C, Huang AJ and Shao ZM: Germline genetic variants disturbing the Let-7/LIN28 double-negative feedback loop alter breast cancer susceptibility. PLoS Genet 7: e1002259, 2011.
13. Zhang J, Zhang L, Fan R, Guo N, Xiong C, Wang L, Jin S, Li W and $\mathrm{Lu} \mathrm{J}$ : The polymorphism in the let-7 targeted region of the Lin28 gene is associated with increased risk of type 2 diabetes mellitus. Mol Cell Endocrinol 375: 53-57, 2013.

14. Johnson AM and Olefsky JM: The origins and drivers of insulin resistance. Cell 152: 673-684, 2013.

15. Veerapur VP, Prabhakar KR, Kandadi MR, Srinivasan KK and Unnikrishnan MK: Antidiabetic effect of Dodonaea viscosa aerial parts in high fat diet and low dose streptozotocin-induced type 2 diabetic rats: A mechanistic approach. Pharm Biol 48: 1137-1148, 2010.

16. Chang HC and Guarente L: SIRT1 and other sirtuins in metabolism. Trends Endocrinol Metab 25: 138-145, 2014.

17. El-Mahmoudy A, Shimizu Y, Shiina T, Matsuyama H,El-Sayed M and Takewaki T: Successful abrogation by thymoquinone against induction of diabetes mellitus with streptozotocin via nitric oxide inhibitory mechanism. Int Immunopharmacol 5: 195-207, 2005.

18. Mondol V and Pasquinelli AE: Let's make it happen: The role of let-7 microRNA in development. Curr Top Dev Biol 99: 1-30, 2012.

19. Thornton JE and Gregory RI: How does lin28 let-7 control development and disease? Trends Cell Biol 22: 474-482, 2012.

20. Frost RJ and Olson EN: Control of glucose homeostasis and insulin sensitivity by the Let-7 family of microRNAs. Proc Natl Acad Sci USA 108: 21075-21080, 2011.

21. Zhang C, Bao W, Rong Y, Yang H, Bowers K, Yeung E and Kiely M: Genetic variants and the risk of gestational diabetes mellitus: A systematic review. Hum Reprod Update 19: 376-390, 2013.

22. Petrie JR, Pearson ER and Sutherland C: Implications of genome wide association studies for the understanding of type 2 diabetes pathophysiology. Biochem Pharmacol 81: 471-477, 2011.

23. Rutanen J, Yaluri N, Modi S, Pihlajamäki J, Vänttinen M, Itkonen P, Kainulainen S, Yamamoto H, Lagouge M, Sinclair DA, et al: SIRT1 mRNA expression may be associated with energy expenditure and insulin sensitivity. Diabetes 59: 829-835, 2010. 